Supporting Information for

\title{
Solid State Chemical Transformations to Enhance Gas Capture in Benzoxazine-Linked Conjugated Microporous Polymers
}

\author{
Mohamed Gamal Mohamed, ${ }^{1}$ Tzu-Chun Chen, ${ }^{1}$ and Shiao-Wei Kuo ${ }^{1,2 *}$
}

${ }^{1}$ Department of Materials and Optoelectronic Science, Center of Crystal Research, National Sun Yat-Sen University, Kaohsiung 80424, Taiwan.

${ }^{2}$ Department of Medicinal and Applied Chemistry, Kaohsiung Medical University, Kaohsiung 807, Taiwan.

\author{
Corresponding author \\ E-mail: kuosw@ffaculty.nsysu.edu.tw
}




\section{Characterization}

FTIR spectra were collected using a Bruker Tensor 27 FTIR spectrophotometer at a resolution of $4 \mathrm{~cm}^{-1}$ and the $\mathrm{KBr}$ disk method. ${ }^{13} \mathrm{C}$ nuclear magnetic resonance (NMR) spectra were recorded using an INOVA 500 instrument, with DMSO as the solvent and tetramethylsilane (TMS) as the external standard; chemical shifts are reported in parts per million (ppm). The molecular weights of the TPE-aniline-Br$r_{4}$ Schiff base, TPE-hydroxybenzylamine- $\mathrm{Br}_{4}$ and $\mathrm{TPE}-\mathrm{BZ}-\mathrm{Br}_{4}$ were determined using a Bruker Solarix high-resolution Fourier transform mass spectrometer (Bruker, Bremen, Germany). The thermal stabilities of the samples under $\mathrm{N}_{2}$ were measured using a TG Q-50 thermogravimetric analyzer; the cured sample (ca. $5 \mathrm{mg}$ ) was placed in a Pt cell and then heated at $20{ }^{\circ} \mathrm{C} \mathrm{min}^{-1}$ from 100 to $800{ }^{\circ} \mathrm{C}$ under a $\mathrm{N}_{2}$ flow of $60 \mathrm{~mL} \mathrm{~min}{ }^{-1}$. Wide-angle X-ray diffraction (WAXD) patterns were measured at the wiggler beamline BL17A1 of the National Synchrotron Radiation Research Center (NSRRC), Taiwan; a triangular bent Si (111) single crystal was used to obtain a monochromated beam having a wavelength $(\lambda)$ of $1.33 \AA$. The morphologies of the polymer samples were examined through field emission scanning electron microscopy (FE-SEM; JEOL JSM7610F) and transmission electron microscopy (TEM), using a JEOL-2100 microscope operated at an accelerating voltage of $200 \mathrm{kV}$. BET surface areas and porosimetry measurements of the samples (ca. 40-100 mg) were performed using a BEL Master $^{\mathrm{TM}}$ instrument and BEL $\operatorname{sim}^{\mathrm{TM}}$ software (v. 3.0.0); $\mathrm{N}_{2}$ adsorption and desorption isotherms were generated through incremental exposure to ultrahigh-purity $\mathrm{N}_{2}$ (up to ca. $1 \mathrm{~atm}$ ) in a liquid $\mathrm{N}_{2}(77 \mathrm{~K})$ bath; surface parameters were calculated using the BET adsorption models in the instrument's software. The pore sizes of the prepared samples were determined using nonlocal density functional theory (NLDFT). 
Table S1. Summarized the thermal stabilities of the TPE-BZ-Br 4 , TPE-TPE-BZ CMP and PyTPE-BZ CMP before and after thermal curing.

\begin{tabular}{cccc}
\hline Sample & $\mathrm{T}_{d 5}\left({ }^{\circ} \mathrm{C}\right)$ & $\mathrm{T}_{d 10}\left({ }^{\circ} \mathrm{C}\right)$ & Char Yield (\%) \\
\hline TPE-BZ-Br & 279 & 314 & 48.2 \\
\hline $180^{\circ} \mathrm{C}$ & 281 & 315 & 49.1 \\
\hline $210^{\circ} \mathrm{C}$ & 316 & 340 & 51.0 \\
\hline $250^{\circ} \mathrm{C}$ & 351 & 364 & 50.4 \\
\hline $280^{\circ} \mathrm{C}$ & 384 & 412 & 52.0 \\
\hline $300^{\circ} \mathrm{C}$ & 406 & 436 & 52.3 \\
\hline TPE-TPE-BZ CMP & 309 & 402 & 68.3 \\
\hline $180^{\circ} \mathrm{C}$ & 353 & 419 & 66.4 \\
\hline $210^{\circ} \mathrm{C}$ & 354 & 413 & 61.7 \\
\hline $250^{\circ} \mathrm{C}$ & 382 & 431 & 62.2 \\
\hline $280^{\circ} \mathrm{C}$ & 413 & 471 & 61.8 \\
\hline $300^{\circ} \mathrm{C}$ & 416 & 475 & 63.0 \\
\hline $350^{\circ} \mathrm{C}$ & 450 & 517 & 63.1 \\
\hline $\mathrm{Py}-\mathrm{TPE}-\mathrm{BZ} \mathrm{CMP}$ & 321 & 407 & 71.2 \\
\hline $180^{\circ} \mathrm{C}$ & 347 & 414 & 71.7 \\
\hline $210^{\circ} \mathrm{C}$ & 350 & 400 & 60.1 \\
\hline $250^{\circ} \mathrm{C}$ & 366 & 411 & 56.2 \\
\hline $280^{\circ} \mathrm{C}$ & 400 & 452 & 55.0 \\
\hline $300^{\circ} \mathrm{C}$ & 436 & 482 & 54.6 \\
\hline $350^{\circ} \mathrm{C}$ & 440 & 495 & 53.7 \\
\hline
\end{tabular}


Table S2. Comparison of TPE-TPE-BZ CMP, Py-TPE-BZ CMP, poly(TPE-TPE-BZ) and poly(Py-TPE-BZ) CMPs with other $\mathrm{N}$-enriched porous carbon materials.

\begin{tabular}{|c|c|c|c|}
\hline \multirow[t]{2}{*}{ Samples } & \multicolumn{2}{|c|}{$\mathrm{CO}_{2}$ uptake (mmole/g) } & \multirow[t]{2}{*}{ Ref } \\
\hline & $298 \mathrm{~K}$ & $273 \mathrm{~K}$ & \\
\hline $\begin{array}{l}\text { TPE-TPE-BZ } \\
\text { CMP }\end{array}$ & 2.18 & 3.3 & This Work \\
\hline Py-TPE-BZ CMP & 1.30 & 1.94 & This Work \\
\hline $\begin{array}{l}\text { poly(TPE-TPE- } \\
\text { BZ) }\end{array}$ & 2.21 & 3.97 & This Work \\
\hline poly(Py-TPE-BZ) & 2.20 & 3.93 & This Work \\
\hline HCM-DAH-1 & 3.30 & - & S1 \\
\hline RLF-500 & 3.13 & & $\mathbf{S 2}$ \\
\hline BPOP-1 & 0.98 & 1.79 & S3 \\
\hline COF-1 & & 1.56 & S4 \\
\hline PTPA-3 & & 1.48 & S5 \\
\hline BOXPOP-1 & & 1.25 & S6 \\
\hline AT-F2-900 & 3.17 & - & S7 \\
\hline NPC-1 & 3.95 & 6.2 & S8 \\
\hline BZPh-A & 1.44 & - & S9 \\
\hline BZPh-CN-A & 2.82 & - & S9 \\
\hline Fc-CMP-1 & & 1.45 & S10 \\
\hline CTHP-3 & 2.07 & 3.21 & S11 \\
\hline A6CMP-3 & - & 3.17 & S12 \\
\hline A6CMP-5 & - & 3.43 & S12 \\
\hline CTF-HUST-3 & - & 3.16 & S13 \\
\hline
\end{tabular}




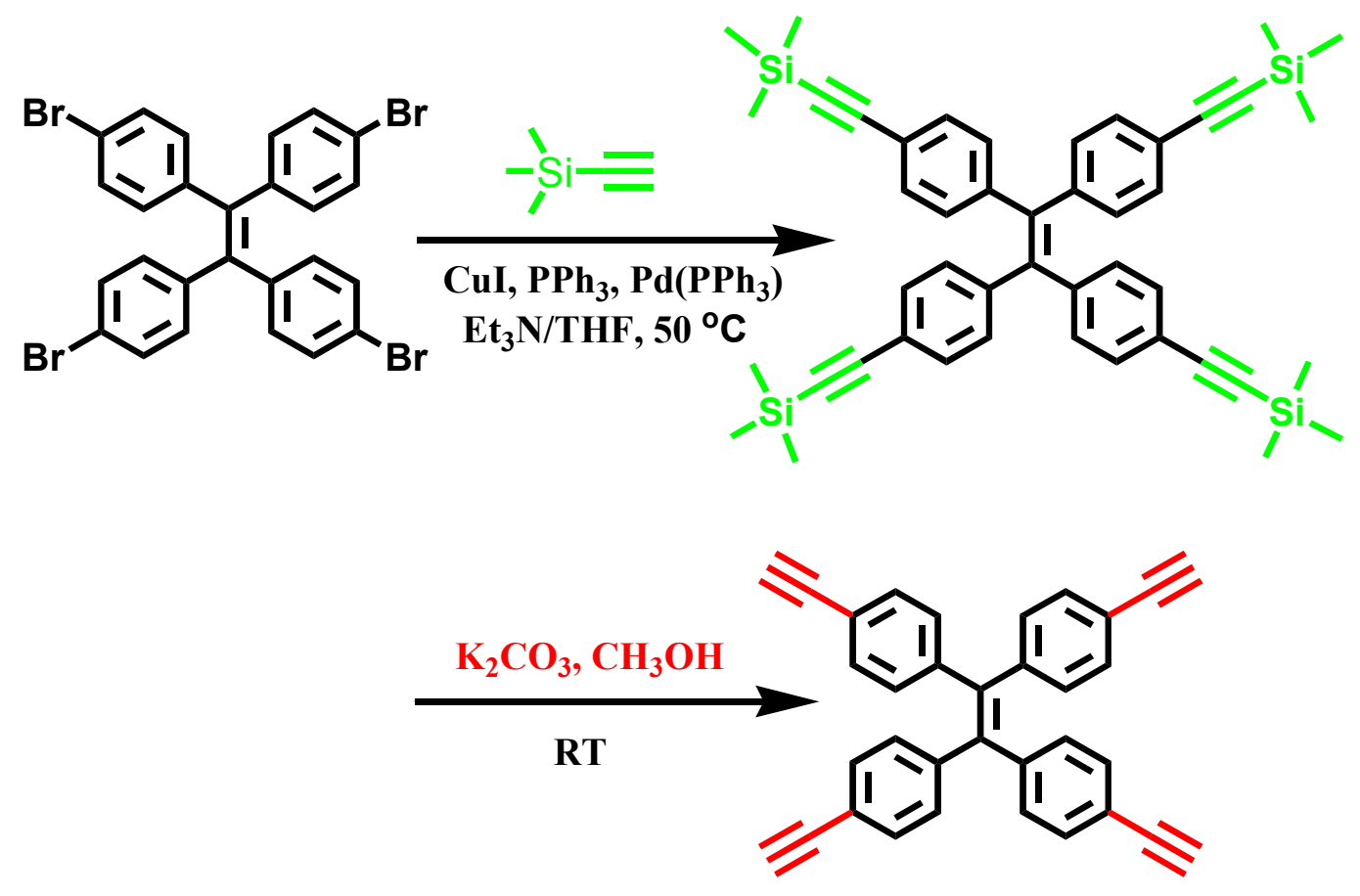

Scheme S1. Synthesis of TEP-T. 


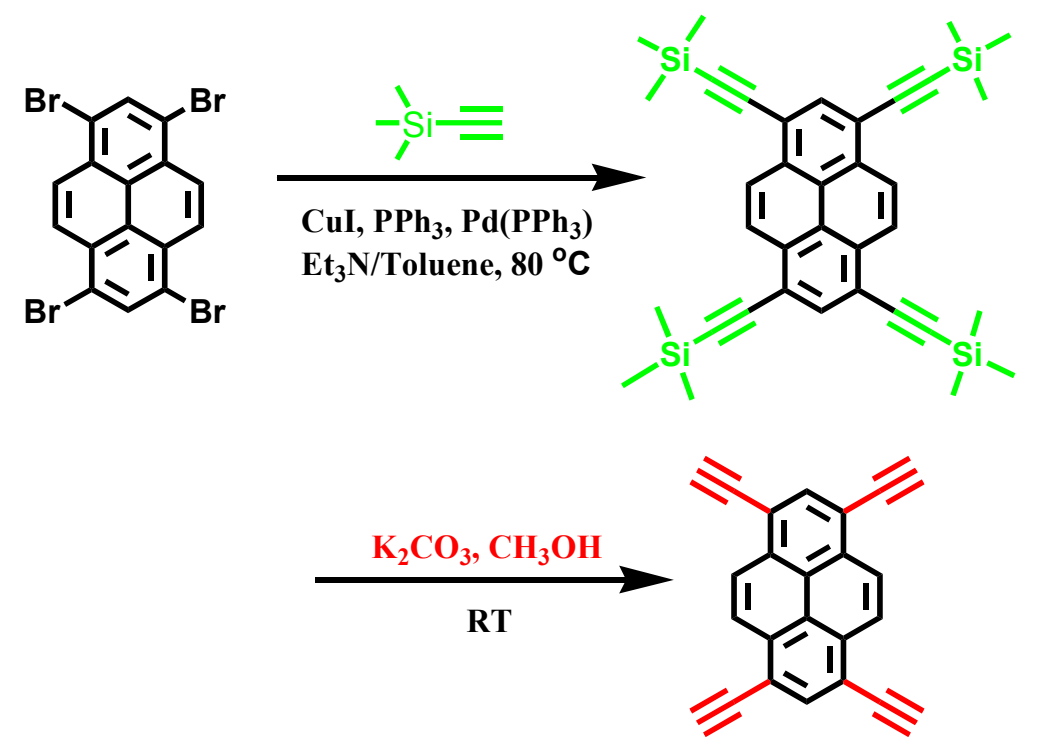

Scheme S2. Synthesis of Py-T. 


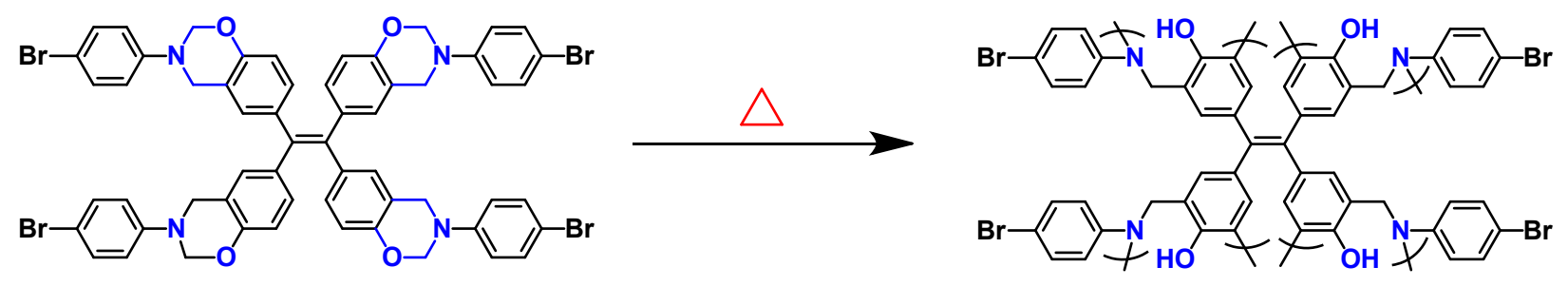

Scheme S3. Ring-opening polymerization of TPE-BZ- $\mathrm{Br}_{4}$ to form poly(TPE-BZ-Br 4 ). 
(a)
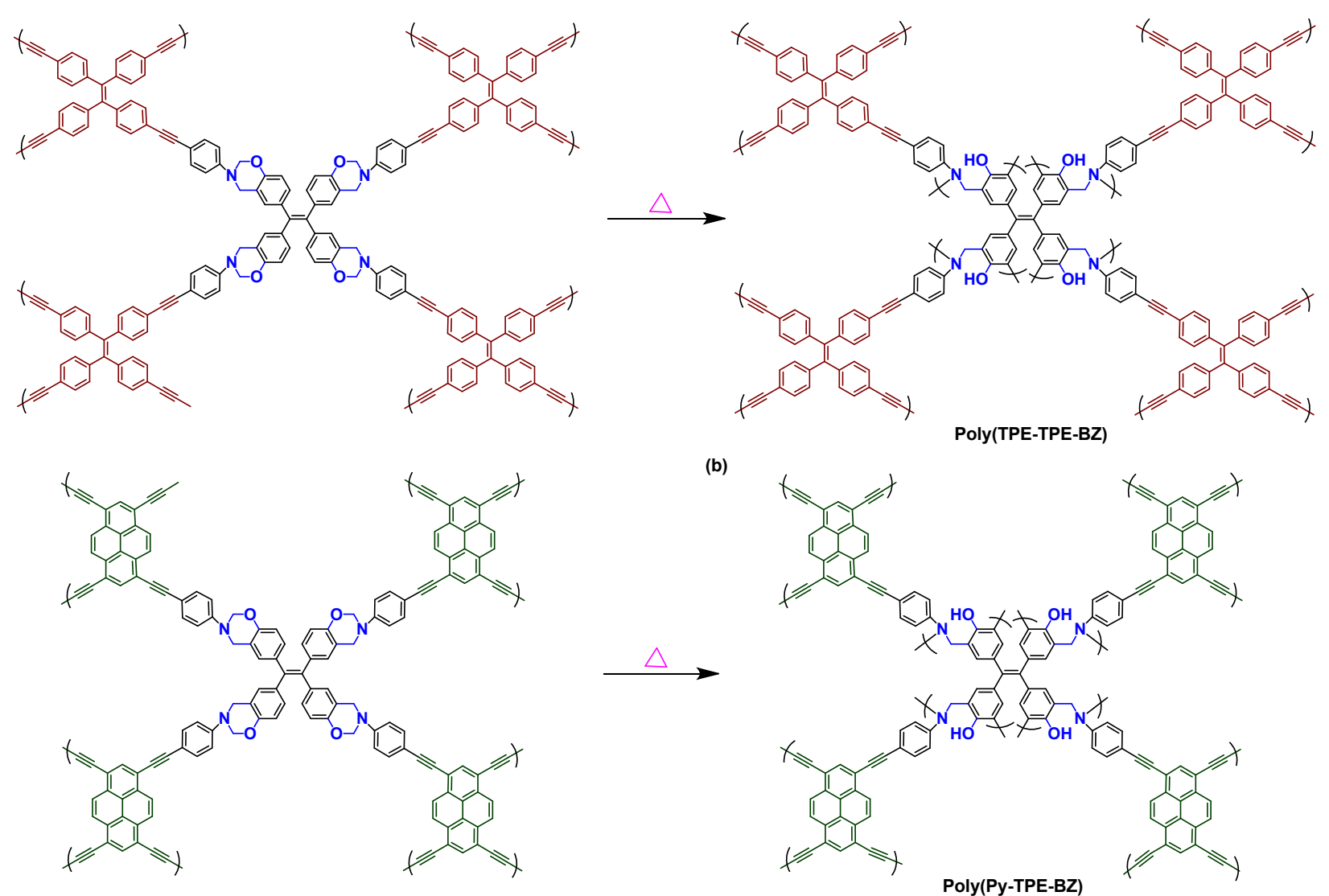

(b)

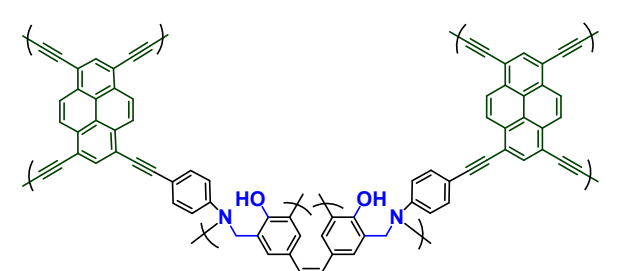

Scheme S4. Ring-opening polymerization of TPE-TPE-BZ CMP and Py-TPE-BZ CMP to form (a) poly(TPE-TPE-BZ) and (b) poly(Py-TPE-BZ) CMPs. 


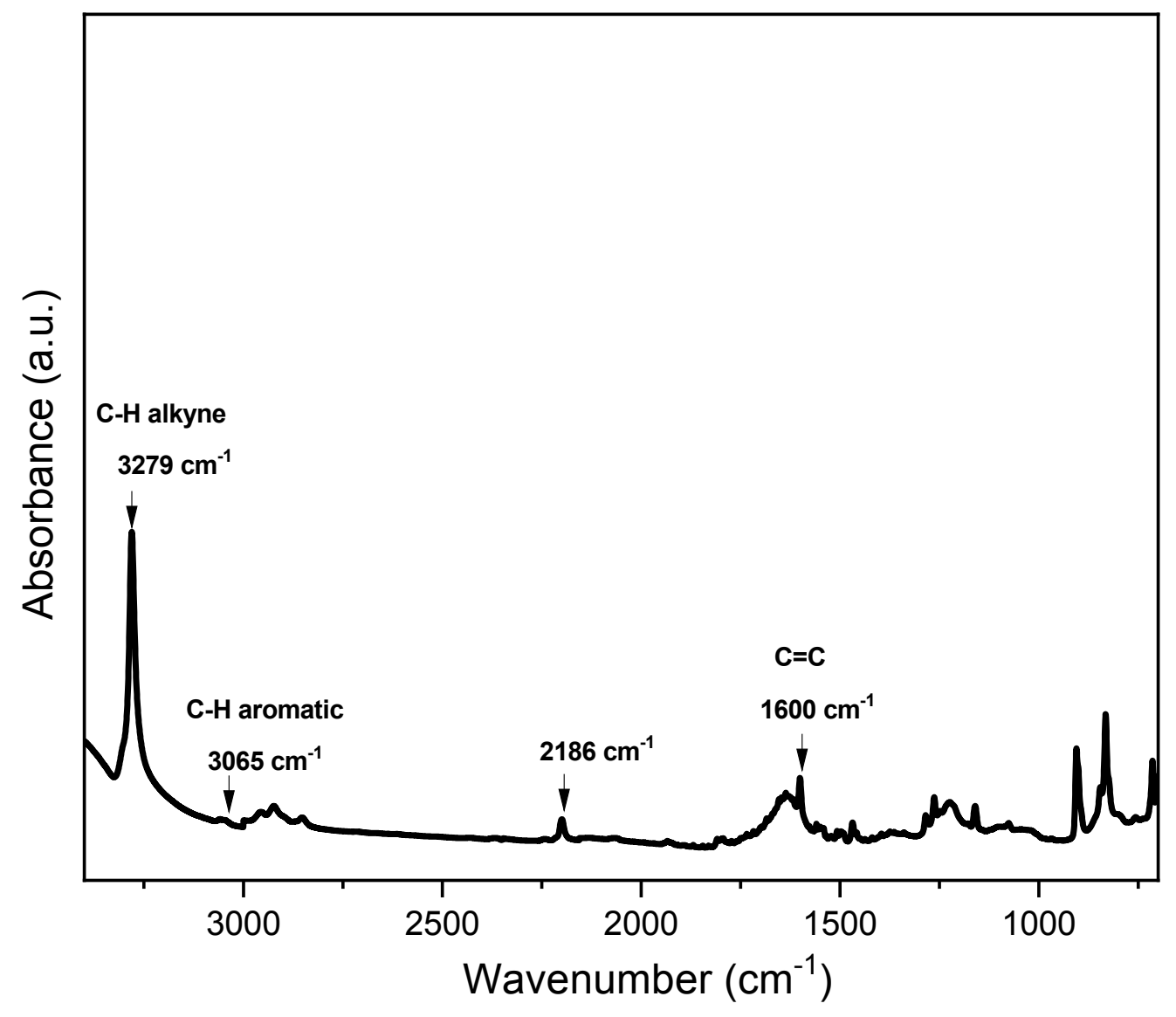

Figure S1. FT-IR spectrum of Py-T. 


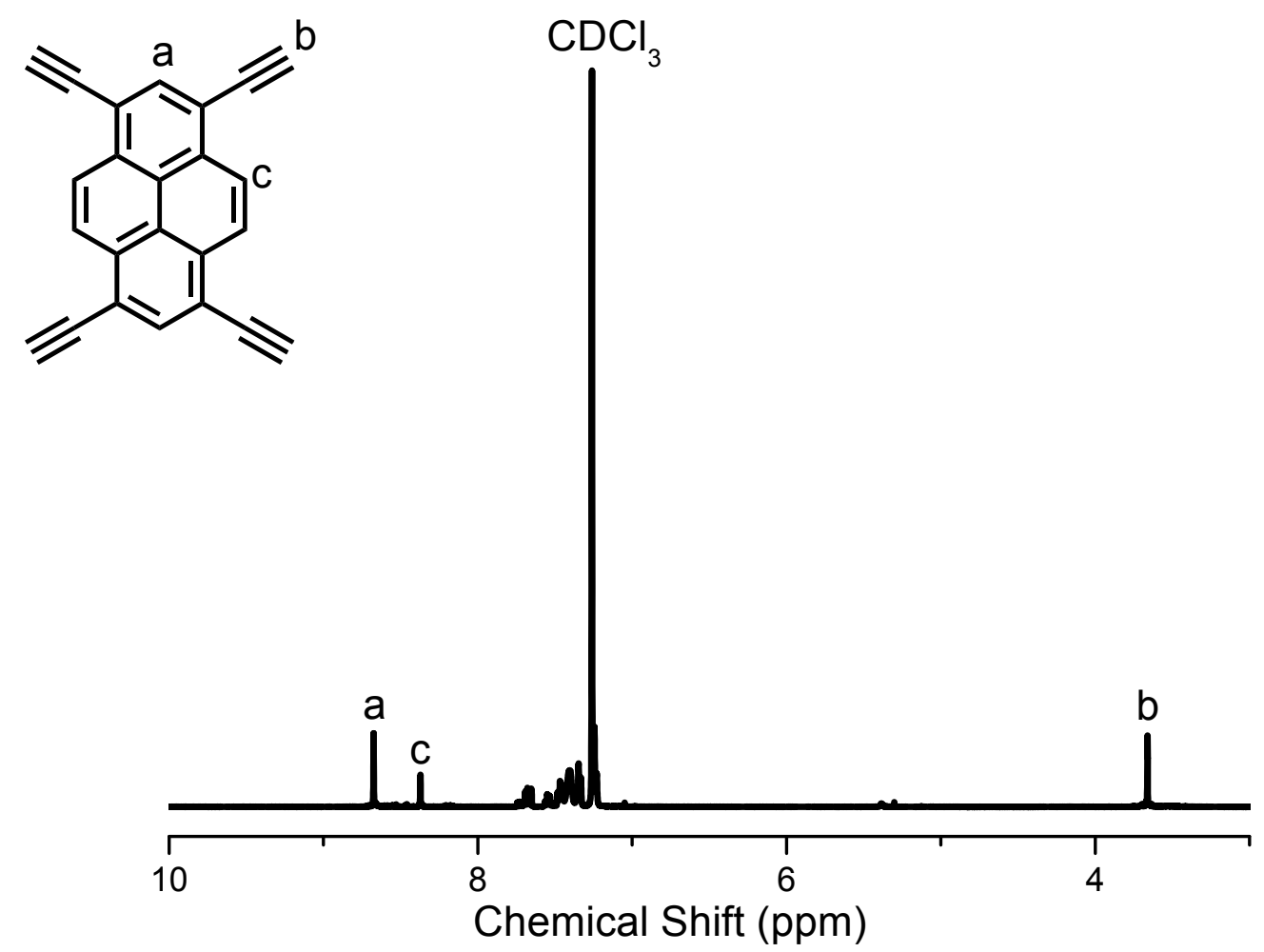

Figure S2. ${ }^{1} \mathrm{H}$ NMR spectrum of Py-T. 


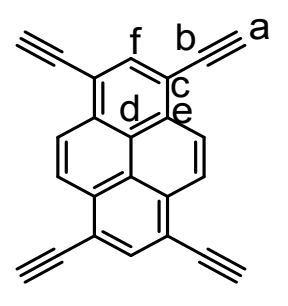

\section{$\mathrm{CDCl}_{3}$}

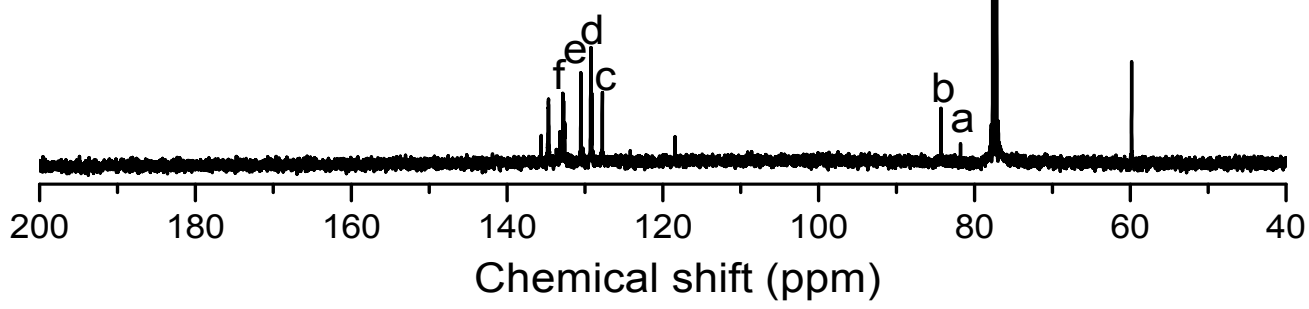

Figure S3. ${ }^{13} \mathrm{C}$ NMR spectrum of Py-T. 


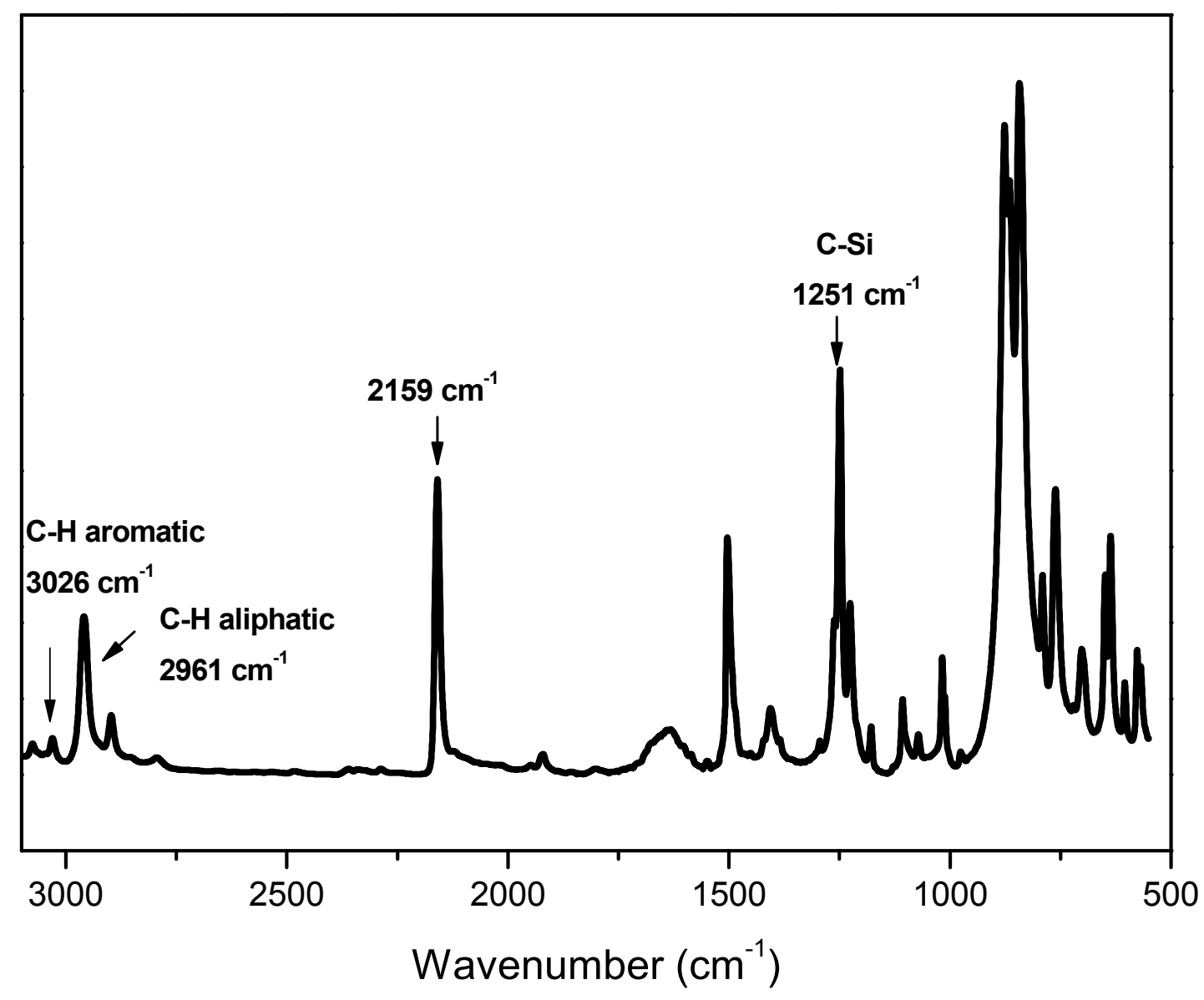

Figure S4. FT-IR spectrum of TPE-TMS. 


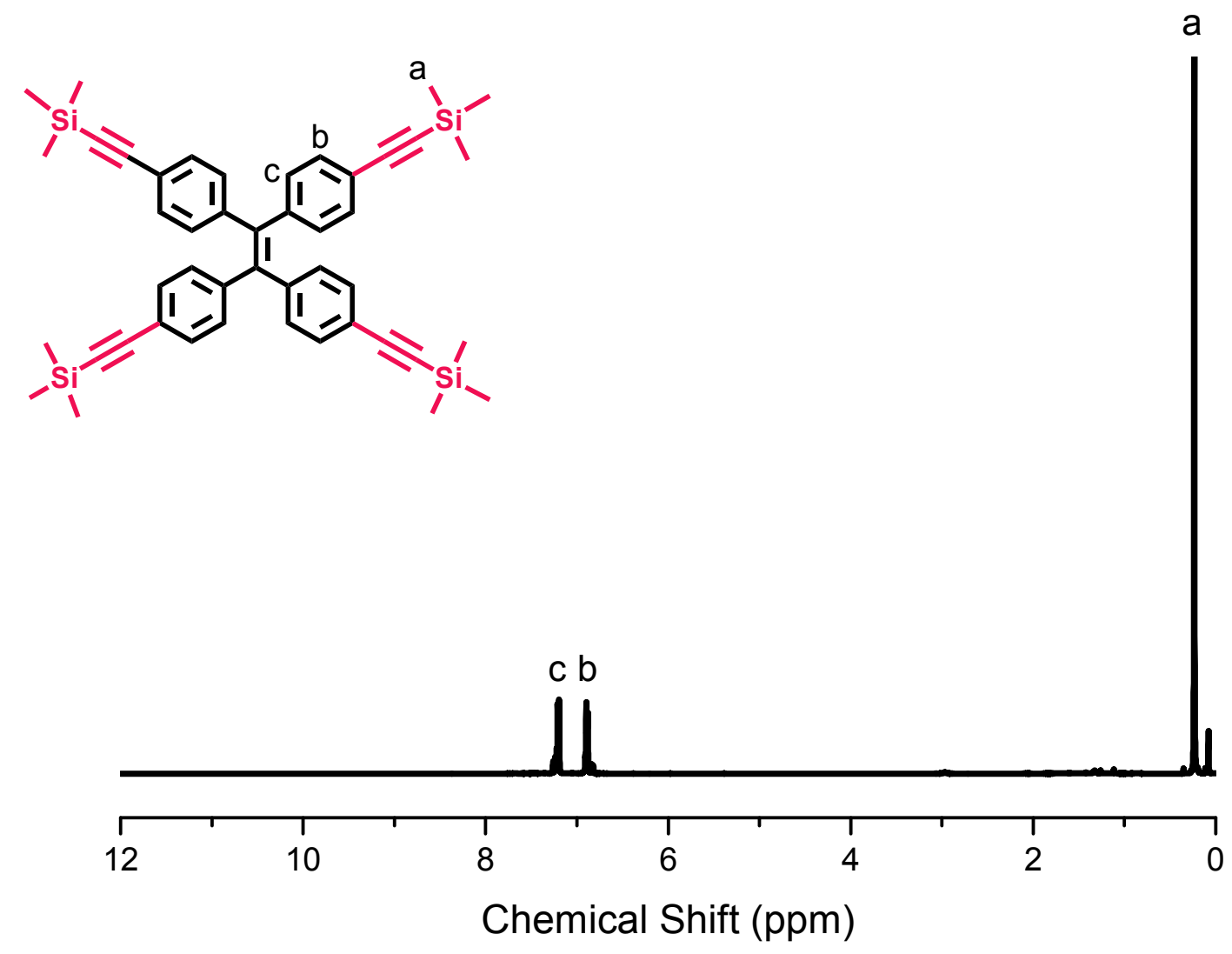

Figure S5. ${ }^{1} \mathrm{H}$ NMR spectrum of TPE-TMS. 


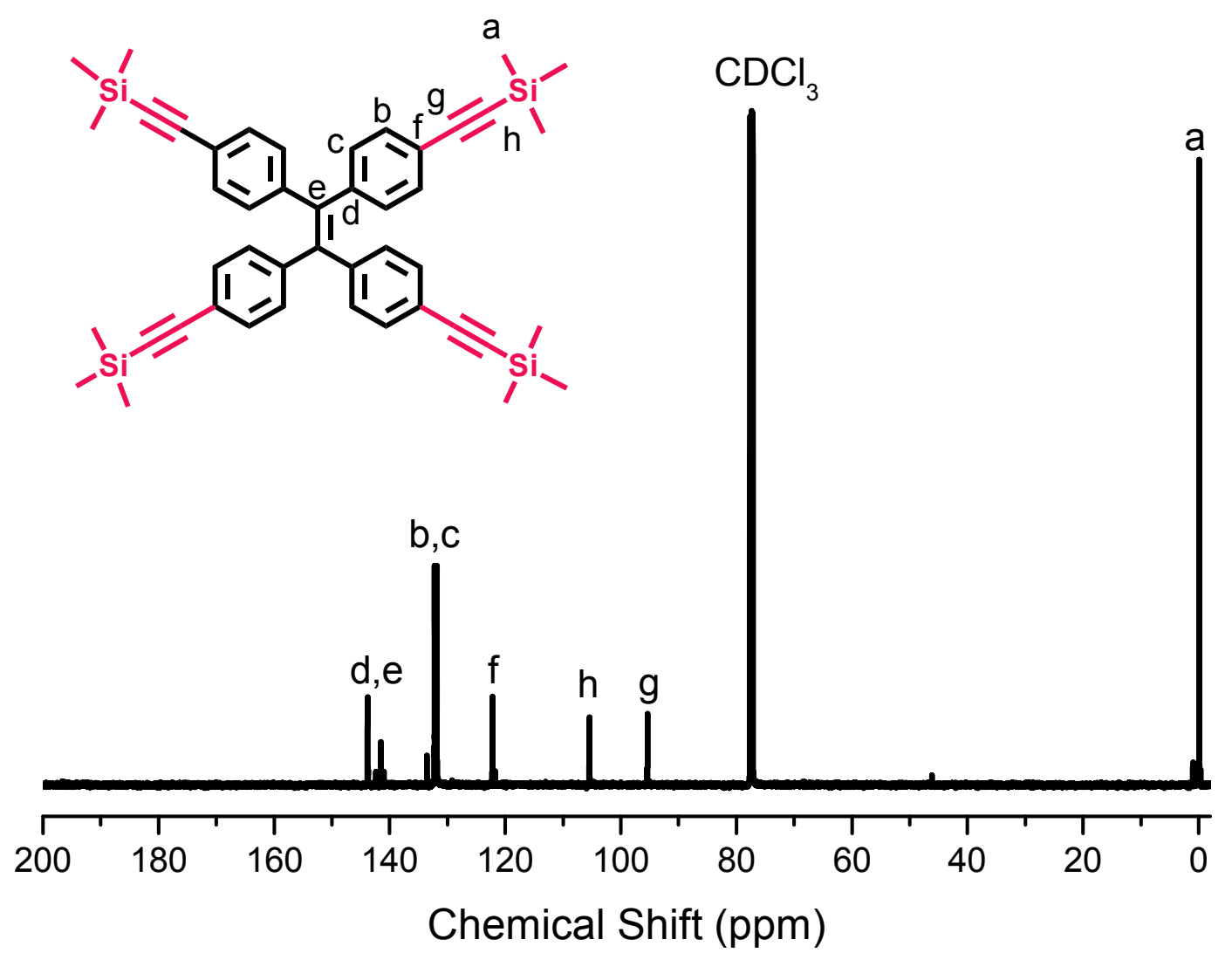

Figure S6. ${ }^{13} \mathrm{C}$ NMR spectrum of TPE-TMS. 


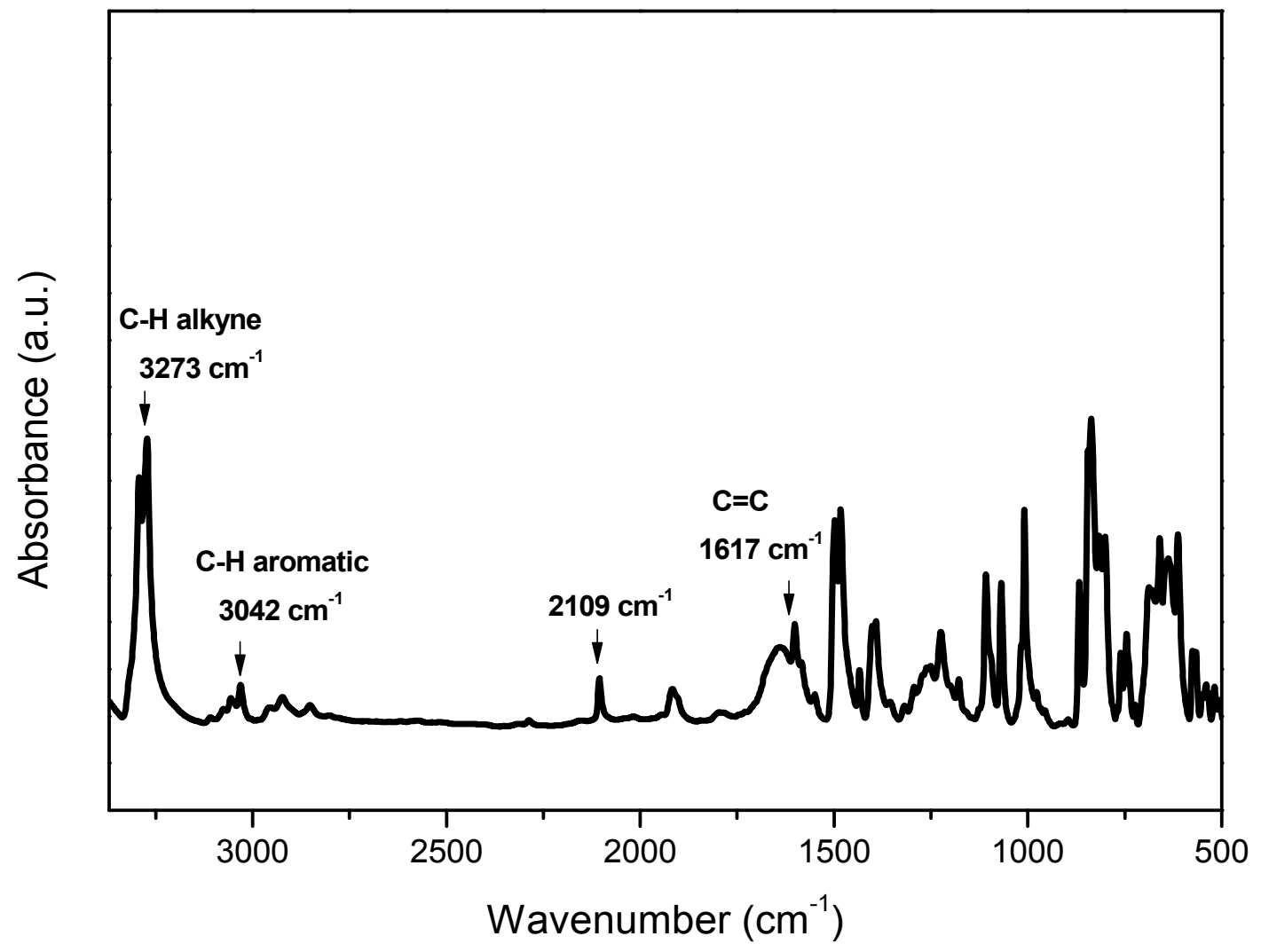

Figure S7. FT-IR spectrum of TPE-T. 


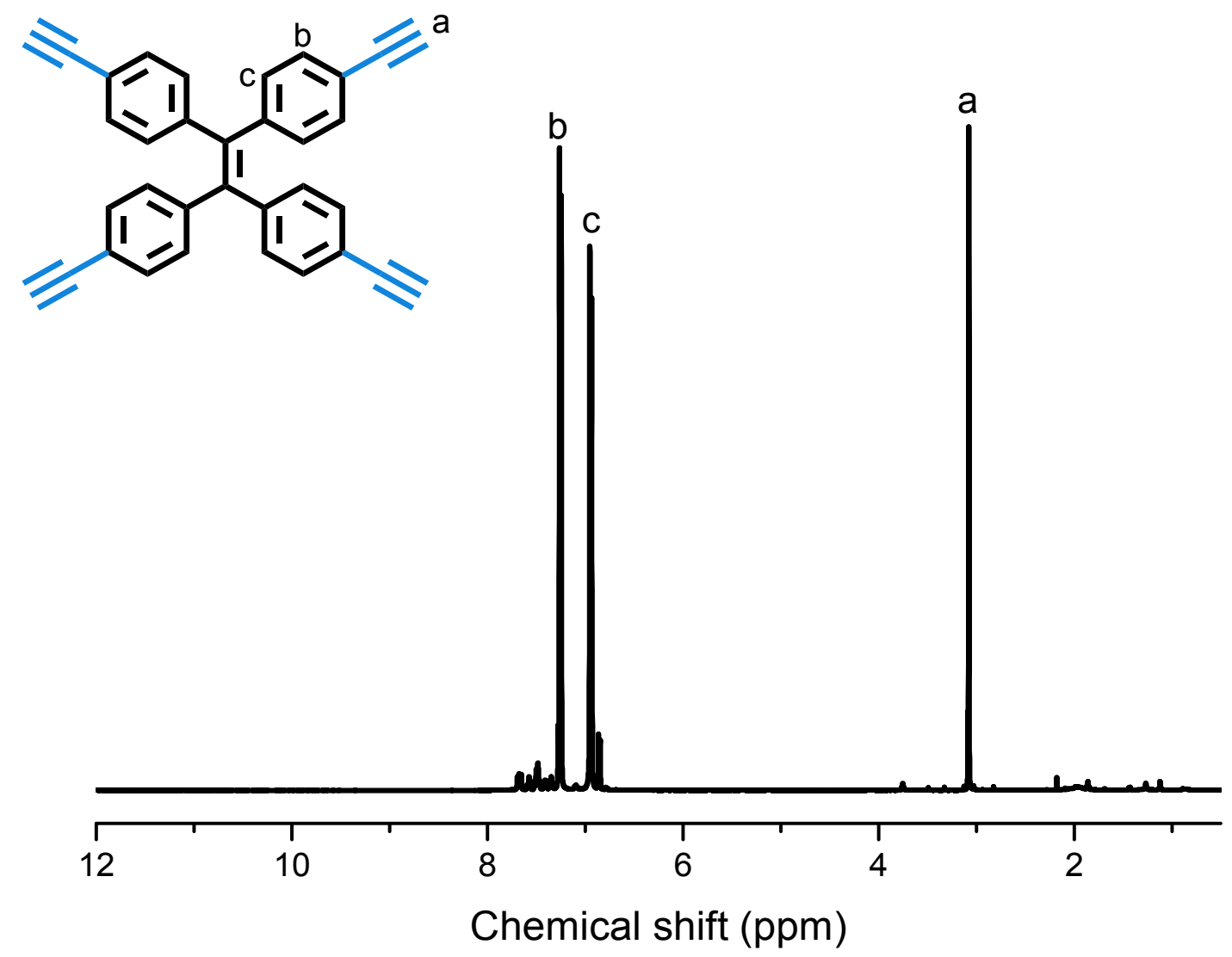

Figure S8. ${ }^{1} \mathrm{H}$ NMR spectrum of TPE-T. 


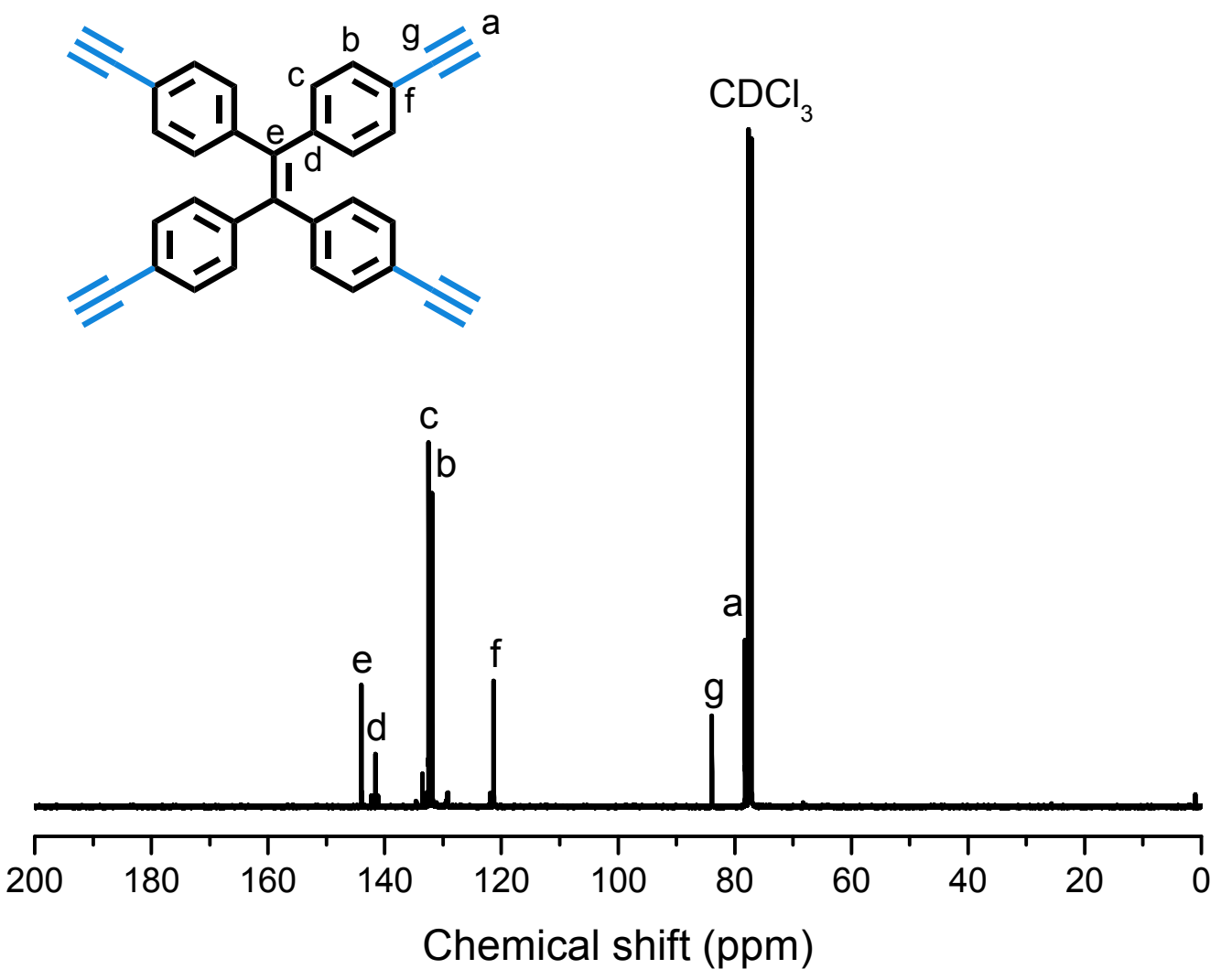

Figure S9. ${ }^{13} \mathrm{C}$ NMR spectrum of TPE-T. 

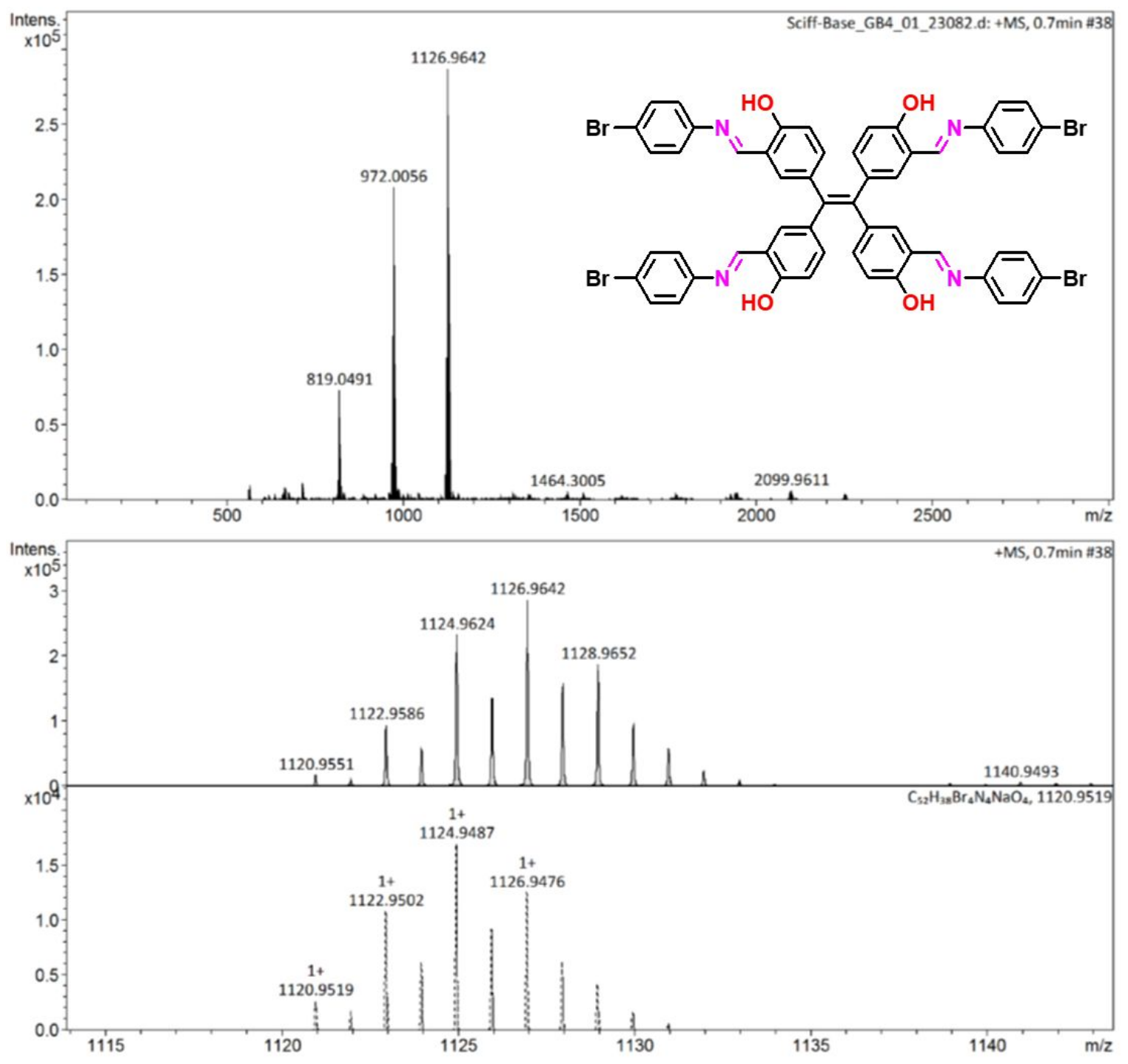

Figure S10. FT mass spectrum of TPE-aniline-Br $\mathrm{Br}_{4}$ Schiff base. 


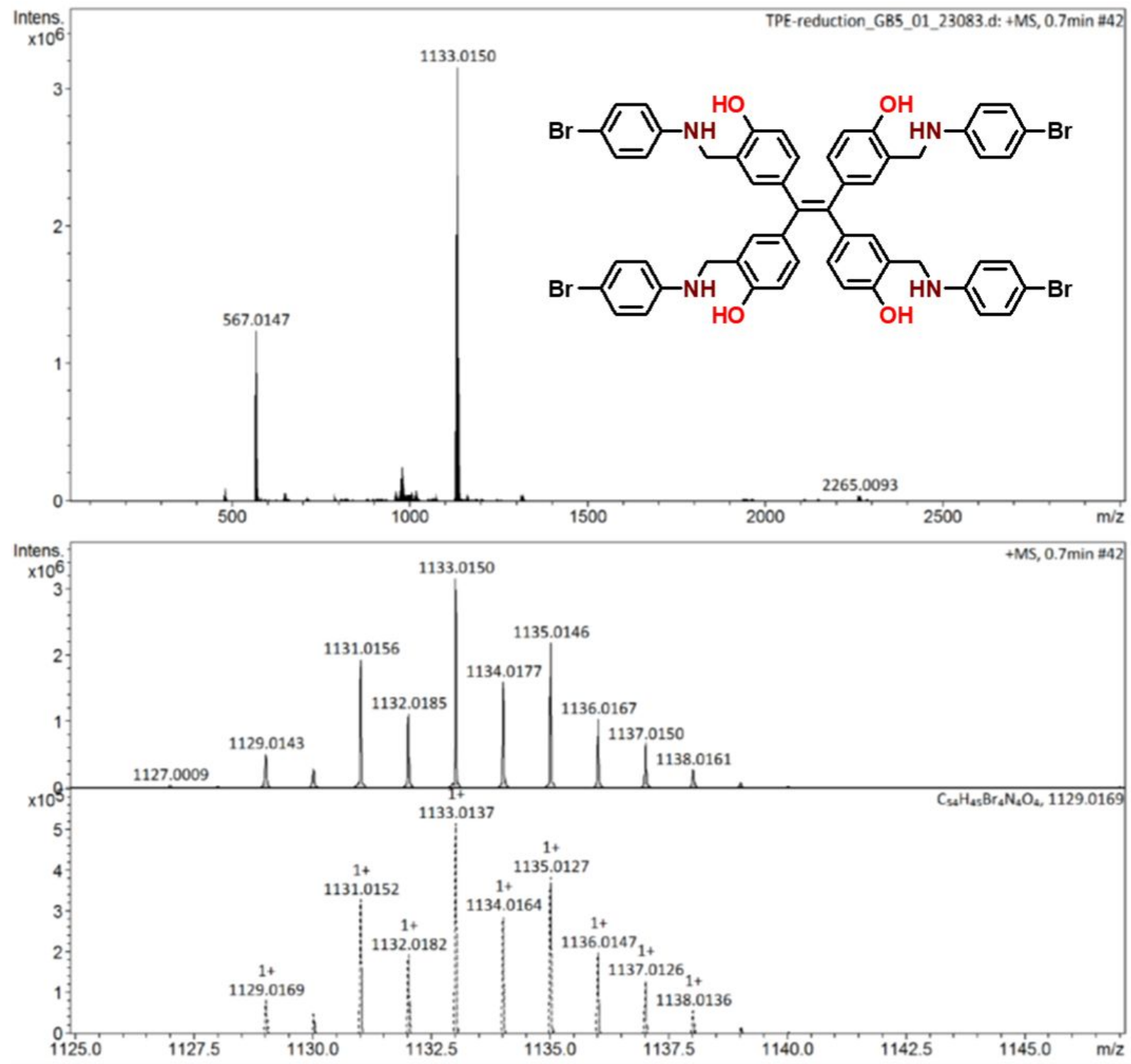

Figure S11. FT mass spectrum of TPE-hydroxybenzylamine- $\mathrm{Br}_{4}$. 

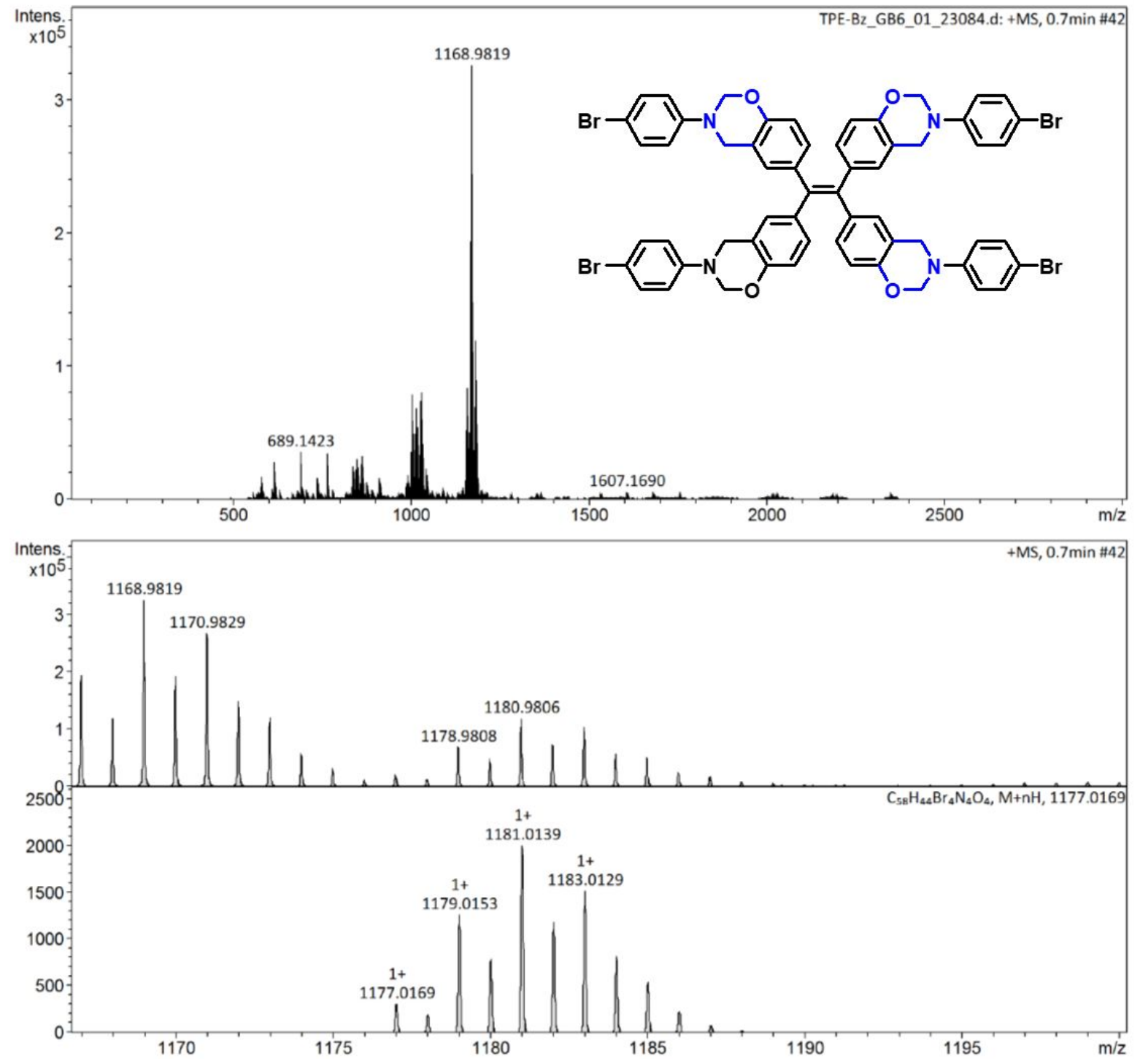

Figure S12. FT mass spectrum of TPE-BZ-Br 4 . 


$$
\ln \left(\frac{\beta}{T_{p}^{2}}\right)=\ln \left(\frac{A R}{E_{a}}\right)-\frac{E_{a}}{R T}
$$

Where $\beta=d T / d t$ is the heating rate, $A$ is the pre-exponential factor, $T_{\mathrm{P}}$ is the exothermic curing peak, and $R$ is the universal gas constant.

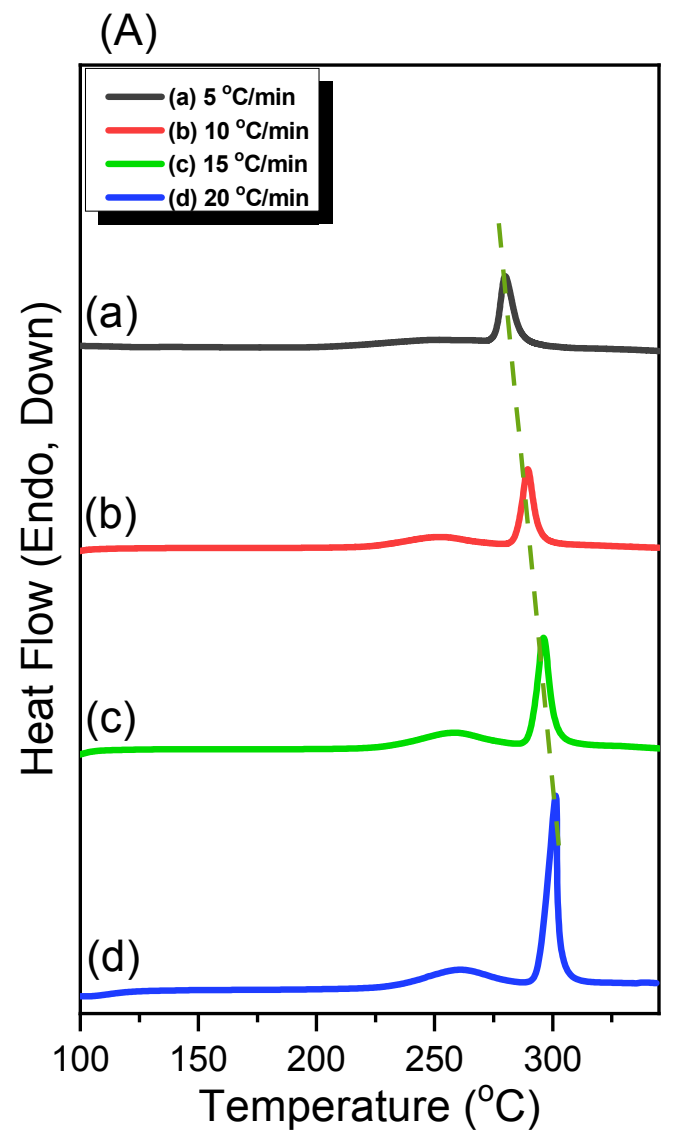

(B)

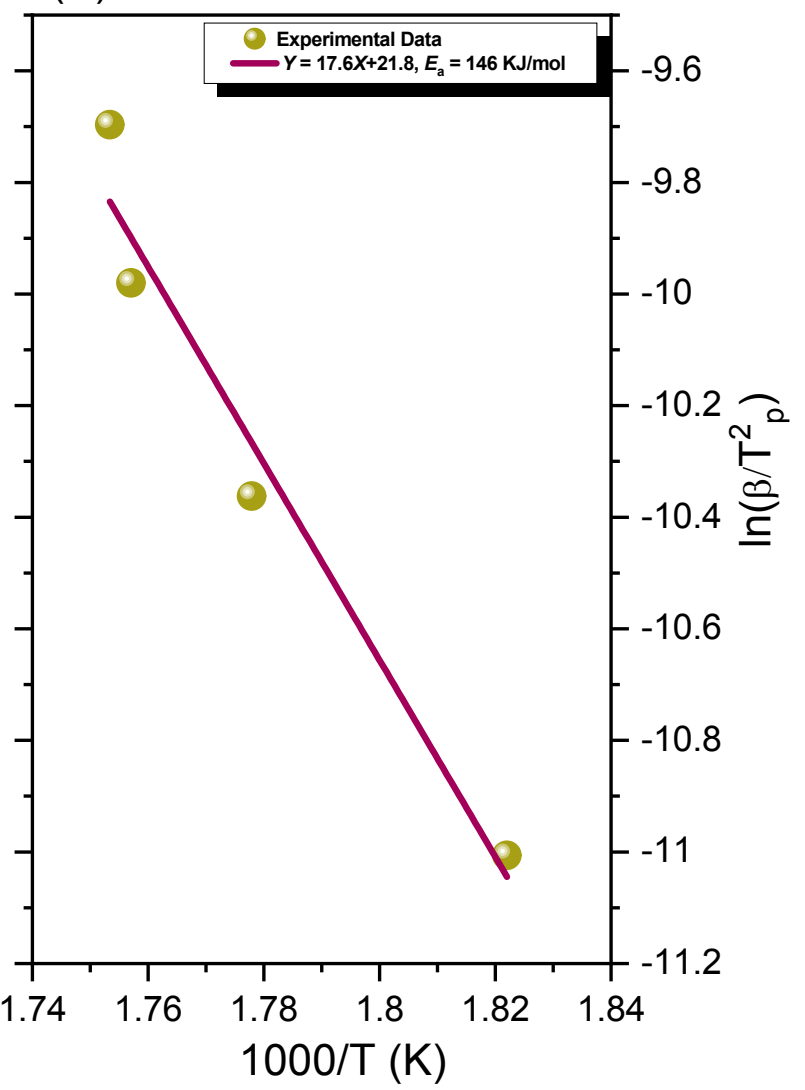

Figure S13. (A) Dynamic DSC exothermic curves and (B) Kissinger plots for determination of $\mathrm{E}_{\mathrm{a}}$ value of pure TPE-BZ-Br${ }_{4}$. 
(a)

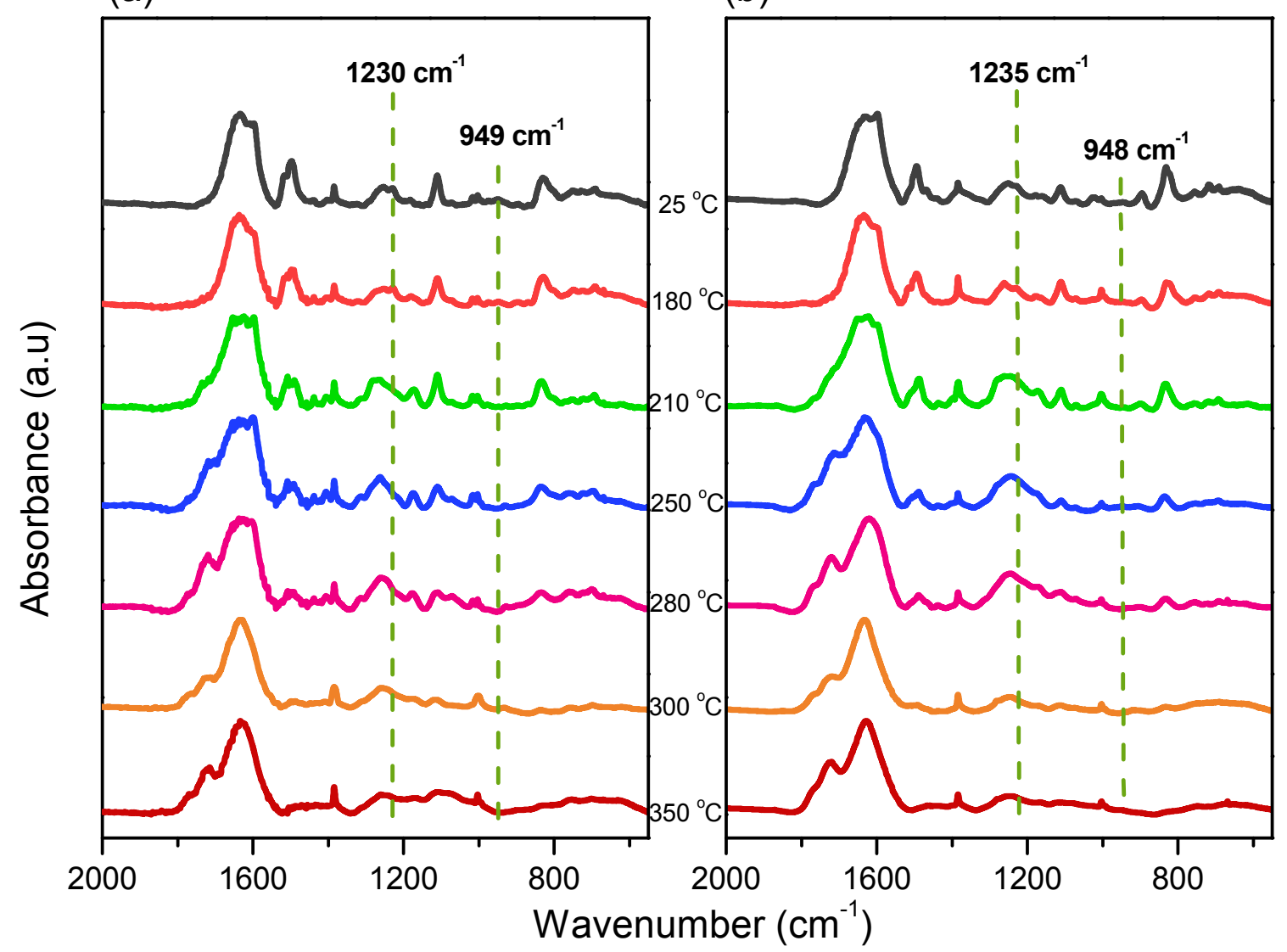

Figure S14. FTIR profiles of (a) TPE-TPE-BZ CMP and (b) Py-TPE-BZ CMP before and after thermal treatments. 


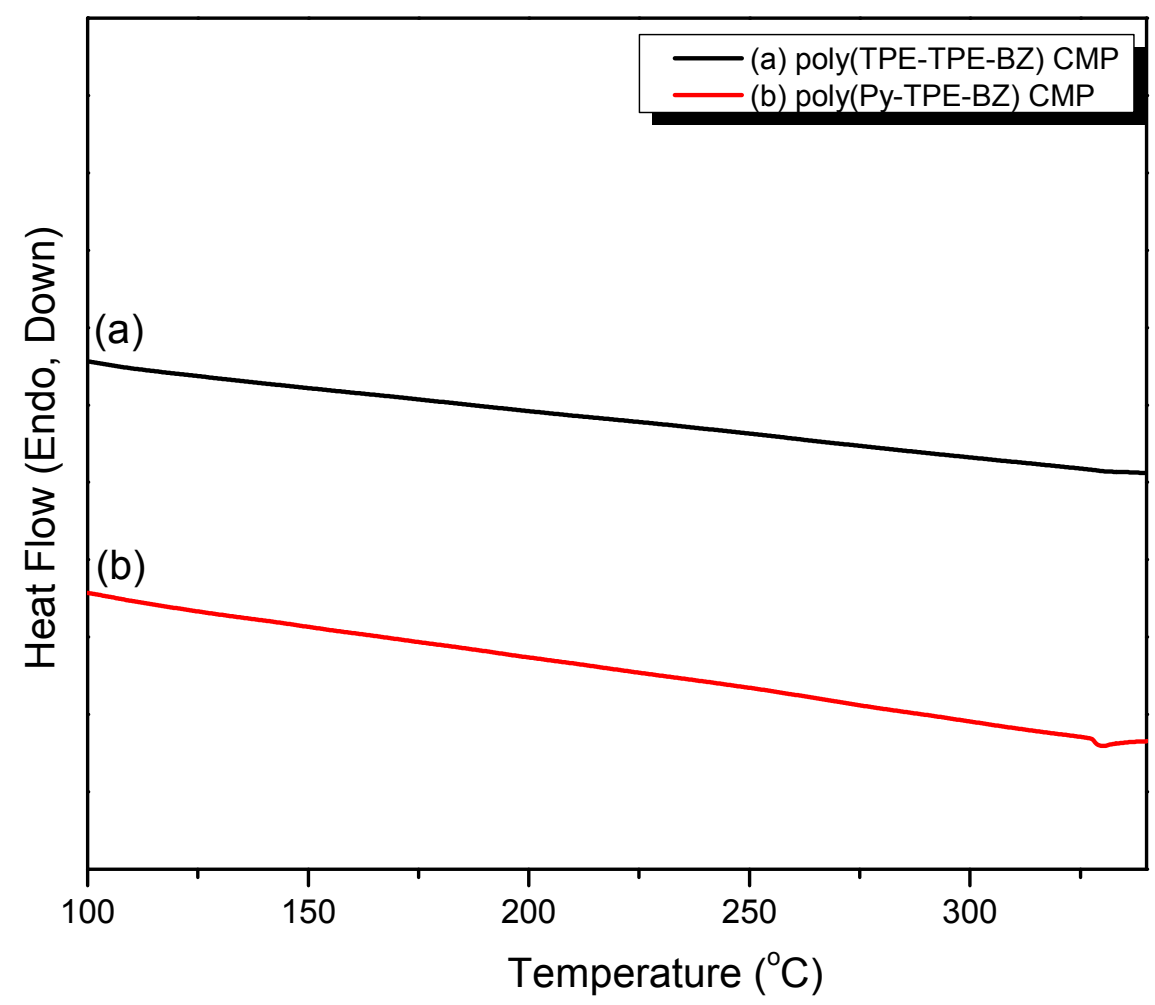

Figure S15. First heating scan of (a) poly(TPE-TPE-BZ) and (b) poly(Py-TPE-BZ) CMPs. 
(a) TPE-TPE-BZ CMP

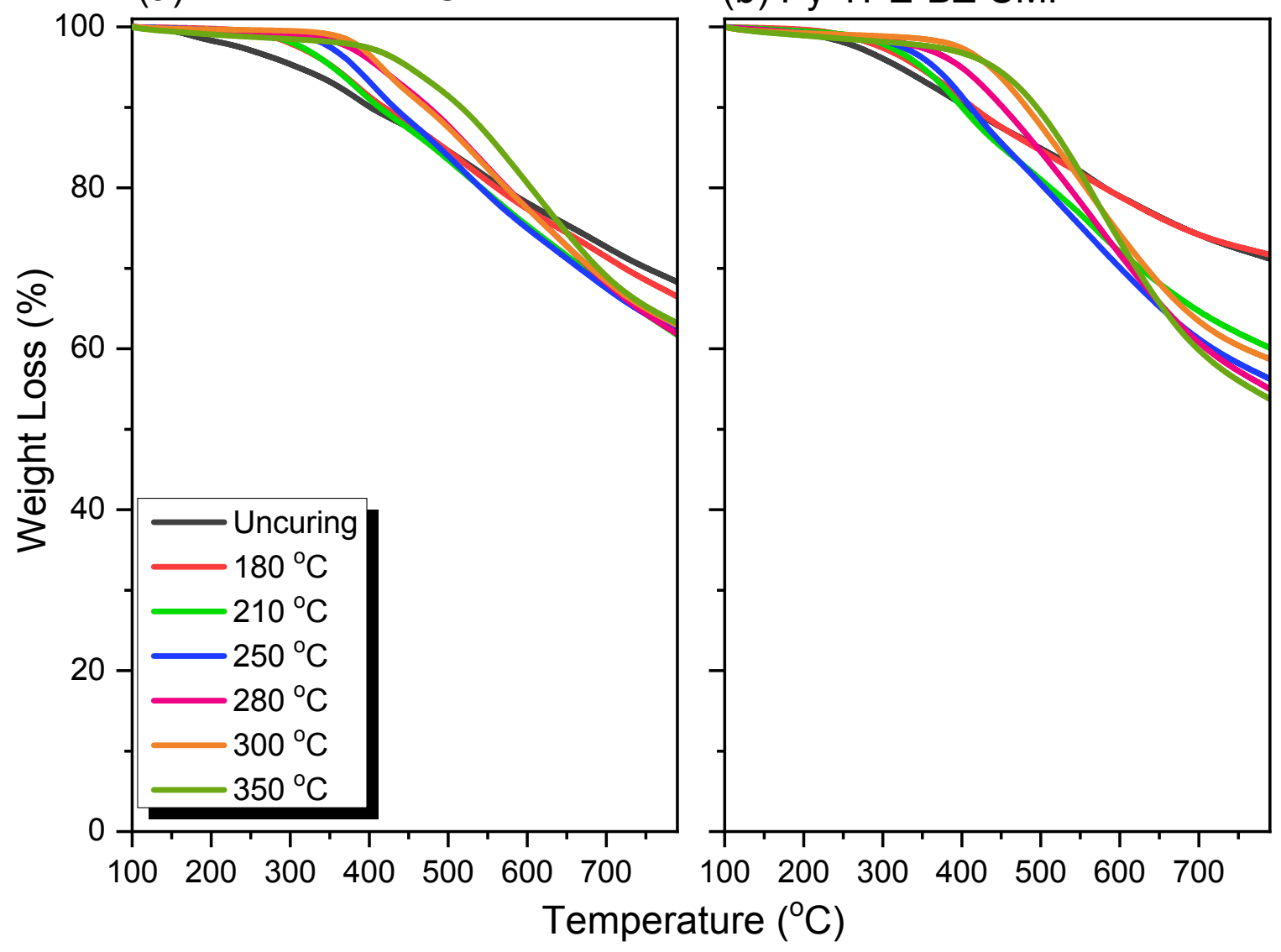

Figure S16. TGA profiles of (a) TPE-TPE-BZ CMP and (b) Py-TPE-BZ CMP before and after thermal treatments. 


\section{References}

[S1] Hao, G. P.; Li , W. C.; Qian , D.; Wang, G. H.; Zhang, W. P.; Zhang, T.; Wang, A. Q.;

Schuth, F.; Bongard, H. J.; Lu, A. H. Structurally Designed Synthesis of Mechanically Stable Poly(benzoxazine-co-resol)-Based Porous Carbon Monoliths and Their Application as HighPerformance $\mathrm{CO}_{2}$ Capture Sorbents. J. Am. Chem. Soc. 2011, 113,11378-11388, Doi.org/10.1021/ja203857g.

[S2] Hao, G. P.; Li, C.; Qian, D.; Lu, A. H. Rapid Synthesis of Nitrogen-Doped Porous Carbon Monolith for $\mathrm{CO}_{2}$ Capture. Adv. Mater.; 2010, 22, 853-857, Doi.org/10.1002/adma.201090014.

[S3] Sun, X.; Li, J.; Wang, W.; Ma, Q. Constructing Benzoxazine-Containing Porous Organic Polymers for Carbon Dioxide and Hydrogen Sorption. Eur. Polym. J. 2018, 107, 89-95, Doi.org/10.1016/j.eurpolymj.2018.07.043.

[S4] Furukawa, H.; Yaghi, O. M. Storage of Hydrogen, Methane, and Carbon Dioxide in Highly Porous Covalent Organic Frameworks for Clean Energy Applications. J. Am. Chem. Soc. 2009, 131, 8875-8883, Doi.org/10.1021/ja9015765.

[S5] Liao, Y.; Weber, J.; Faul, C. F. J. Conjugated Microporous Polytriphenylamine Networks. Chem. Commun. 2014, 50, 8002-8005, Doi.org/10.1039/C4CC03026E.

[S6] Xu, S.; He, J.; Jin, S.; Tan, B. Heteroatom-Rich Porous Organic Polymers Constructed by Benzoxazine Linkage with High Carbon Dioxide Adsorption Affinity. J. Colloid Interface Sci. 2018, 509, 457-462, Doi.org/10.1016/j.jcis.2017.09.009.

[S7] Konnola, R.; Anirudhan, T. S. Efficient Carbon Dioxide Capture by Nitrogen and Sulfur Dual-Doped Mesoporous Carbon Spheres from Polybenzoxazines Synthesized by A Simple Strategy. J. Environ. Chem. Eng. 2020, 8, 103614-103624, Doi.org/10.1016/j.jece.2019.103614. 
[S8] Wan, L.; Wang, J.; Sun, Y.; Feng, C.; Li, K. Polybenzoxazine-Based Nitrogen-Containing Porous Carbons for High-Performance Supercapacitor Electrodes and Carbon Dioxide Capture. RSC Adv, 2015, 5, 5331-5342, Doi.org/10.1039/C4RA13637C.

[S9] Wu, J. Y.; Mohamed, M. G.; Kuo, S. W. Directly Synthesized Nitrogen-Doped Microporous Carbons from Polybenzoxazine Resins for Carbon Dioxide Capture. Polym. Chem, 2017, 8, 5481-5489, Doi.org/10.1039/C7PY01026E.

[S10] Li, G.; Liu, Q.; Liao, B.; Chen, L.; Zhou, H.; Zhou, Z.; Xia, B.; Huang, J.; Liu, B. Synthesis of Novel Ferrocene-Based Conjugated Microporous Polymers with Intrinsic Magnetism. Eur. Polym. J. 2017, 93, 556-560, Doi.org/10.1016/j.eurpolymj.2017.06.034.

[S11] Gao, H.; Ding, L.; Bai, H.; Li, L. Microporous Organic Polymers Based on HyperCrosslinked Coal Tar: Preparation and Application for Gas Adsorption. ChemSusChem 2017, 10, 618-623, Doi.org/10.1002/cssc.201601475.

[S12] Qin, L.; Xu, G.; Yao, C.; Xu, Y. Conjugated Microporous Polymer Networks with Adjustable Microstructures for High $\mathrm{CO}_{2}$ Uptake Capacity and Selectivity. Chem Commun 2016, 52,12602-12605, Doi.org/10.1039/C6CC05097B.

[S13] Wang, K.; Yang, L.; Wang, X.; Guo, L.; Cheng, G.; Zhang, C.; Jin, S.; Tan, B.; Cooper, A. Covalent Triazine Frameworks via A Low Temperature Polycondensation Approach. Angew. Chem. Int. Ed. 2017, 56, 14149-14153, Doi.org/10.1002/anie.201708548. 